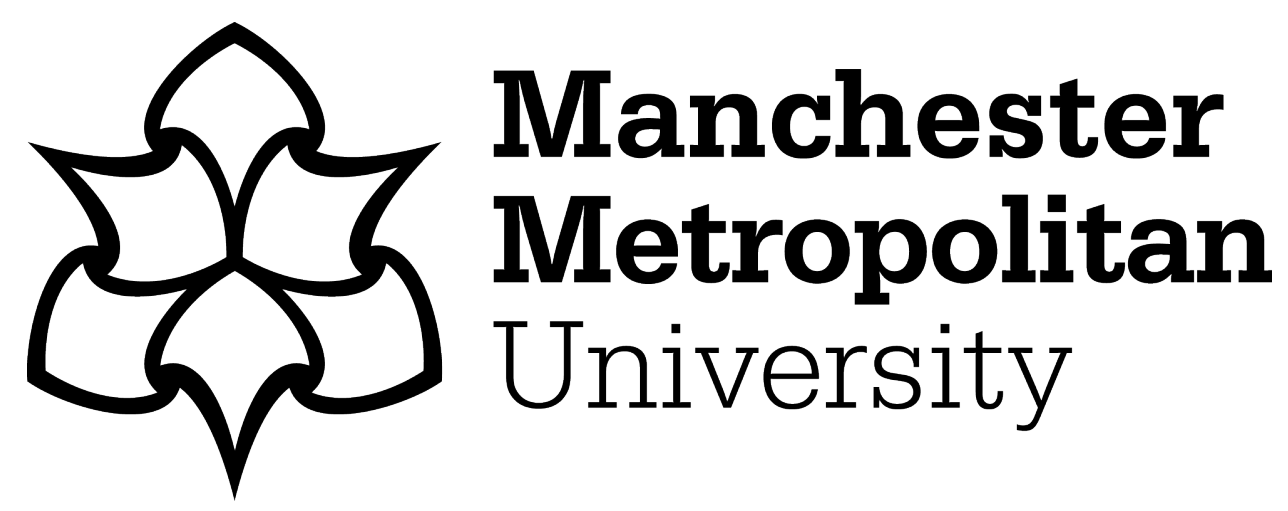

Southgate, M and Bowring, N (2014) Investigation of a Portable Real Time Radiation Mapper. In: 9th International Symposium on Communication Systems, Networks and Digital Signal Processing, CSNDSP 2014, 23 July 2014 - 25 July 2014, Manchester, UK.

Downloaded from: https://e-space.mmu.ac.uk/621315/

Version: Accepted Version

Publisher: IEEE

DOI: https://doi.org/10.1109/CSNDSP.2014.6923991

Please cite the published version 


\title{
Investigation of a Portable Real Time Radiation Mapper
}

\author{
M. J. Southgate and N. J. Bowring \\ Manchester Metropolitan University, School of Engineering, Manchester, UK \\ m.southgate@mmu.ac.uk
}

\begin{abstract}
The naked eye is capable of perceiving electromagnetic radiation in a narrow band of frequencies, known as the optical spectrum. Working with low energy radiation outside the optical spectrum presents distinct difficulties, requiring the use of apparatus to measure invisible and intangible electromagnetic radiation. The apparatus, incorporating a Wiimote, and associated method are presented for a small, easily maintained and operated, low cost system for the characterisation of practical RF radiation sources in the microwave and millimetre-wave frequency bands.
\end{abstract}

Keywords - 3D localisation; computer vision; object tracking; radio frequency.

\section{INTRODUCTION}

Electromagnetic (EM) radiation above and below the visible frequency band, $430-790 \mathrm{THz}$, requires apparatus to translate invisible and intangible energy into an interpretable form. Research into and methods of remotely detecting concealed threats make extensive use of microwave and mm-wave radiation at frequencies above $1 \mathrm{GHz}$ and below $300 \mathrm{GHz}$ [1-3]. Systems capable of generating images at these frequencies are large, expensive and often contain complex optics [4,5]. The feasibility of a lightweight, low cost, intuitively operated apparatus is realised based on a previous idea [6] for the real time visualisation of EM field intensity and mapping. The realisation of the idea consists of readily available components.

Applications include beam pattern and side lobe profiling; verification of a manufactured radiation source against a software model; and assisted alignment of radiation sources and optics during laboratory based EM experiments by inclusion of a video overlay displaying radiation intensity.

Challenges to the development of the system were primarily due to the design constraint that low cost and readily available parts should be used. The final apparatus satisfies these criteria resulting in a system that can be cheaply and easily implemented.

This paper is organised as follows. Section 2 will

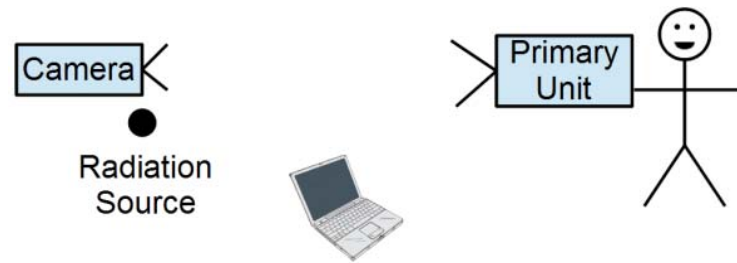

Figure 1. Apparatus and Experimental Setup review the individual hardware and software elements of the apparatus. Section 3 describes the theory of operation for the apparatus and decisions affecting operational performance. Section 4 outlines the practical use of the system to gather microwave intensity data and functional limitations of the design. Section 5 presents a practical evaluation of system resolution and examples of microwave intensity maps generated in real-time with a discussion on the effects of data filtering and receiver sensitivity. Lastly, Section 6 summaries the system and its novel features.

\section{APPARATUS}

The apparatus and the intended experimental setup are shown in Fig. 1. The apparatus consists of the Primary Unit (PU) for measuring and processing radiation data, an imaging device to enable spatial determination of the PU and a computer to store and present the data in the desired format.

The PU is designed to interface with a series of interchangeable RF front ends allowing the measurement of a different frequency bands; circuitry to amplify and condition the detected signal; a microcontroller for signal acquisition and basic signal processing; a local GUI displays the current measurement GUI and a wireless link for sending processed data to the computer. All of these operations are performed in real-time at high video rate speeds. The Primary Unit may be used alone as a dumb radiation meter providing instantaneous measurements.

The Camera may be any video camera capable of connecting to the Computer. To achieve an acceptable spatial accuracy a high resolution of $1024 \times 768$ or better is required. The rate of data collection is directly proportional to the video frame rate. When selecting a video source resolution had the greatest impact upon performance. After attempts to use a standard RGB webcam the infrared (IR) camera of WiiMote was tested and found to satisfy this need. An added advantage of the onboard hardware is integrated signal processing for blob detection and the convenience of a Bluetooth connection.

The Computer is a $\mathrm{PC}$ running a $\mathrm{C \#}$ application which populates a point cloud with radiation intensity data from the PU. The position of the PU and hence the location within the point cloud, is determined by examination of the PU in the video imagery generated by the Camera and the transformation of its virtual $2 \mathrm{D}$ video coordinates into real world $3 \mathrm{D}$ coordinates. The application performs all of these operations in real-time. The software, written in $\mathrm{CH}$ uses the Open Source EMGU CV [7] wrapper for the computer vision library OpenCV [8] and WiimoteLib [9].

The prototype uses a Monolithic microwave integrated circuit (MMIC) RF front end with embedded amplifier 
sensitive to radiation in the $75-110 \mathrm{GHz}$ frequency band and an infrared camera with an image resolution of $1024 \times 768$ and frame rate of $100 \mathrm{~Hz}$. The cost of apparatus, excluding the MMIC, is less than $£ 50$.

\section{THEORY OF OPERATION}

\section{A. Overview}

The PU is held in the hand and moved within the Field Of View (FOV) of the Camera. When activated the PU continuously measures, processes and displays radiation intensity data before sending it to the Computer. Simultaneously the Computer is processing imagery from the Camera to determine the real world location of the PU to populate a $3 \mathrm{D}$ point cloud of intensity measurements for the radiation source under characterisation.

\section{B. Primary Unit (PU)}

The PU continuously measures the RF flux intensity at a point in space. The signal from the interchangeable detector is conditioned before digitisation by a microcontroller ADC of 10-bit resolution at $10 \mathrm{kHz}$. By oversampling the signal above the Nyquist rate an improvement in Signal to Noise Ratio (SNR) can be achieved for uncorrelated noise.

The SNR gain due to oversampling is expressed in (1):

$$
S N R_{O S}=10 \log \left(\frac{f S}{2 B}\right)
$$

where $f_{S}$ is the sample rate and $B$ is the highest modulated frequency of the signal. For example, a radiation source with a maximum modulated frequency of $100 \mathrm{~Hz}$ gives an improvement of system noise of $17 \mathrm{~dB}$.

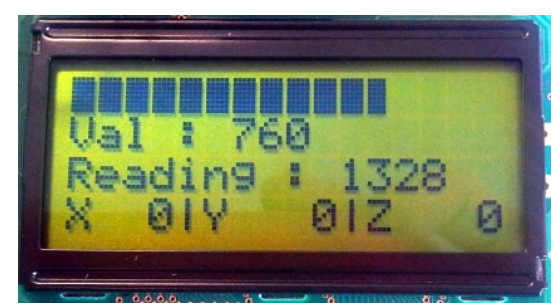

Figure 2. Primary Unit display showing the current measurement value, reading number and spatial position

The processed intensity is displayed on the local LCD, see Fig. 2, with other operating parameters before transmission to the Computer.

\section{Tracking and Localisation of the Primary Unit}

Video generated by the Camera contains spatial information about the Primary Unit. For successfully tracking of the PU it must be distinctive and for localisation, the camera must be calibrated.

Approaches to object tracking were investigated including chroma keying and Speeded Up Robust Feature (SURF) tracking [10]. Each approach failed to perform satisfactorily due to the lab environment and eventually simple blob detection using two infrared light emitting diodes (LEDs) mounted on the PU was tried and selected. This approach was found to provide the best approach in all respects, requiring minimal CPU time, a high refresh

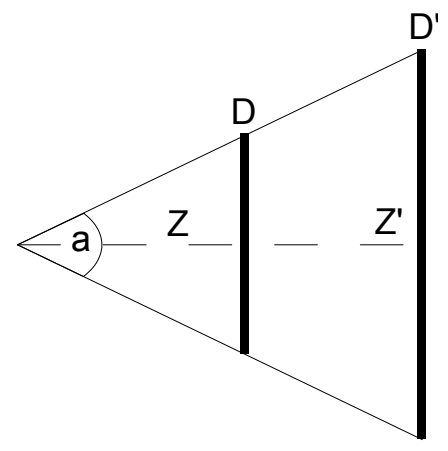

Figure 3. Similar triangles and the apparent size of an object in an image as a function of distance

rate and tracking that is virtually immune to misidentification of the target object.

For accurate measurements the camera must be calibrated using an object of known size and structure. The PU is the perfect object for this task because it is the object to be tracked and its size and shape are known.

The calibration process determines the relationship between what appears on the image and the real world position of the object.

The camera used for tracking the PU has a fixed focus. According to the rule of similar triangles the distance to the PU can be calculated as illustrated in Fig. 3 and expressed in (2):

$$
\frac{D}{Z}=\frac{D^{\prime}}{Z^{\prime}}
$$

where $D$ is a known dimension, $Z$ is a known distance from the camera and $a$ is a fixed angle describing the Field Of View (FOV). This ratio must be maintained if an object at $Z^{\prime}$ is to appear to be the same size $D^{\prime}$.

Therefore as the PU is moved away from the Camera, its virtual size in pixels reduces as $D$ is equal to $D$ '. The software uses an open-source library by Brian Peek [8] to communicate with the camera.

Fig. 4 shows the prototype PU without the LCD module with a wired USB connection for communications and power. The radiation sensitive device shown is a W-band 75-110GHz MMIC LNA and detector.

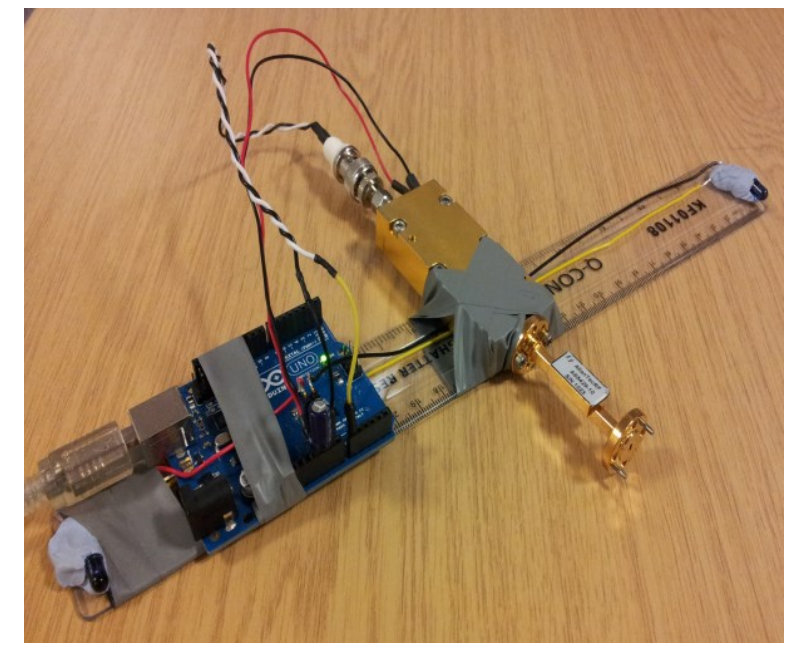

Figure 4. Prototype Primary Unit without local LCD module 


\section{METHOD}

Fig. 1 shows the intended experimental setup. The point cloud data in its raw form is with respect to the camera, so typical practice is to position the camera with the radiation source, but this not an operational requirement. The PU is then moved by hand in the space of interest while automatic data collection occurs. Characterisation of the radiation source is complete when a sufficient number of points have been gathered.

The importance of holding the PU normal to the camera was anticipated during design and proven during experiment. By twisting the PU an incorrect distance is generated by the positioning algorithm with the effect of the PU appearing to be further away than it really is see Fig. 5.

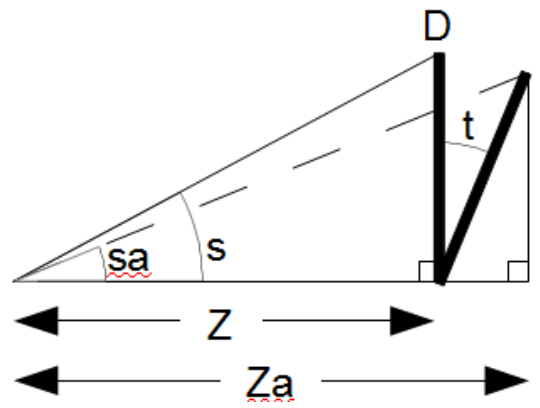

Figure 5. The effect of twisting the Primary Unit upon its calculated distance to the camera

The angle subtended in the FOV of the camera by a twisted PU is reduced from $s$ to $s a$. This has the effect of decreasing the separation of the corner tracking points and increasing the apparent distance $Z$ to the camera by a factor $d_{f}$ to $\mathrm{Za} \mathrm{(3):}$

$$
d_{f}=\frac{1}{\cos t}
$$

where $t$ is the twist angle of the PU. This effect is cumulative in both horizontal and vertical planes; therefore a PU twisted in both planes by $20^{\circ}$ will appear to be $13.2 \%$ further away than it is in reality.

Originally two tracking points in the horizontal plane were used, by increasing this number to four in a square arrangement and averaging the distance calculation of both planes improved performance. A complementary approach limiting the maximum twist was investigated using narrow beam infrared light sources. By limiting the angle with which the tracking points can be seen and discarding data without positional information, a limit can be placed on the effect of twist. The ideal light source to achieve this would be a laser, but the inherent danger of using multiple invisible lasers and the difficulty of maintaining alignment meant that focused LEDs with a beam divergence of $25^{\circ}$ were used instead.

Fig. 6 shows the prototype being used to map the radiation of a known source in a plane at distance $7.5 \mathrm{~m}$. The IR camera is positioned on the radiation source, a black box with a white lens, seen in the distance on the right hand side of the figure. The computer GUI is used during the process of mapping to show the operator areas that have yet to be measured.

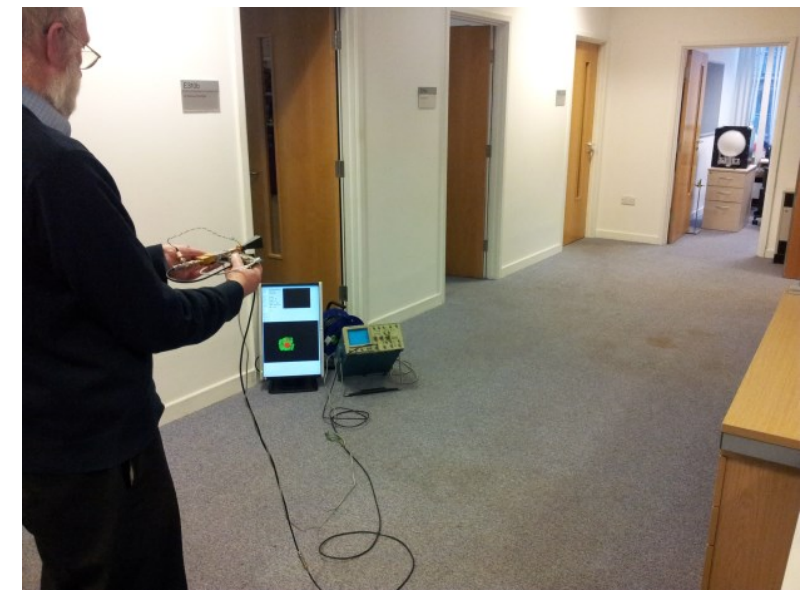

Figure 6. Tethered PU used to measure the radiation pattern of a known radiation source

\section{RESULTS}

Fig. 7 shows the empirical data for a calibration target with differently spaced tracking points at a range of distances. The product of pixel separation and distance described by (2) is satisfied for every point with a maximum error of $2.5 \%$.

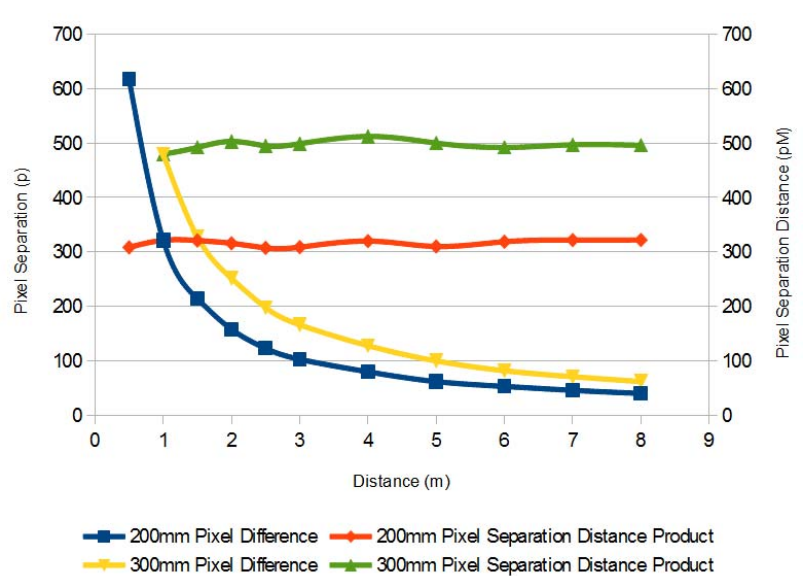

Figure 7. The effect of tracking point spacing (200 and 300mm) against distance as seen by the infrared camera

At distances of less than one metre it was found the field of view of the IR camera was a limitation of the system resulting in difficulty positioning the $300 \mathrm{~mm}$ target so that both tracking points could be seen. This is why the $0.5 \mathrm{~m}$ data point for the $300 \mathrm{~mm}$ target is not shown.

Fig. 8 shows a map of data point coloured according to a threshold for the radiation source shown on the right in Fig. 6. The radiation source is actually a concealed guns and explosives detector with a working range of $25 \mathrm{~m}$ [11]. The active scanner was configured to produce continuous wave radiation of approximate power $10 \mathrm{~mW}$ at $75 \mathrm{GHz}$. The generated radiation is focused through a polyethylene lens by a $15 \mathrm{~dB}$ horn antenna. The directionality of the PU detector was improved using a $20 \mathrm{~dB}$ horn and can be seen in Fig. 6.

The threshold level was set to $80 \%$ of the fully saturated output of the detector. Measurements above the threshold are mapped in red and signals below are shown in green to a position on the plot corresponding to the 


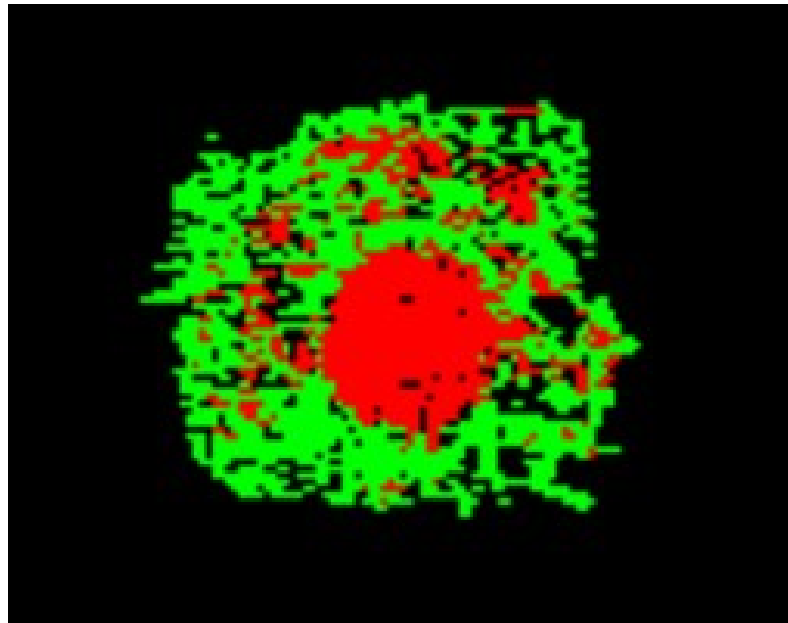

Figure 8. A thresholded radiation map of the MiRTLE at $7.5 \mathrm{~m}$ using a $20 \mathrm{~dB}$ horn antenna to increase PU directionality

location of the PU in the plane of measurement. The black areas of Fig. 8 indicate no valid measurements have been taken at those positions. The range of the measurement plane to the radiation source and the colocated IR camera was used to check for valid measurements, automatically save them and update the radiation map. Measurements not taken in the correct plane are discarded. Subsequent valid measurements are compared with the previous data point for a given location and only the highest is saved and displayed.

The resulting map suffers from surrounding fringes due to the relative ease of which the MMIC detector could be saturated due to the increased sensitivity resulting from the use of a receiver antenna.

Fig. 9 shows a comparison map of the same apparatus, but without a horn antenna on the PU detection element. This reduces the gain of the detector and hence improves the dynamic range by reducing instances of detector saturation for the detected power level.

The resulting map more closely reflects the expected beam pattern and does not suffer from the fringing effects which are reduced below the threshold as seen in Fig. 7. The set of data points on the measurement plane were measured to be $1.5 \mathrm{~m}$ wide as indicated.

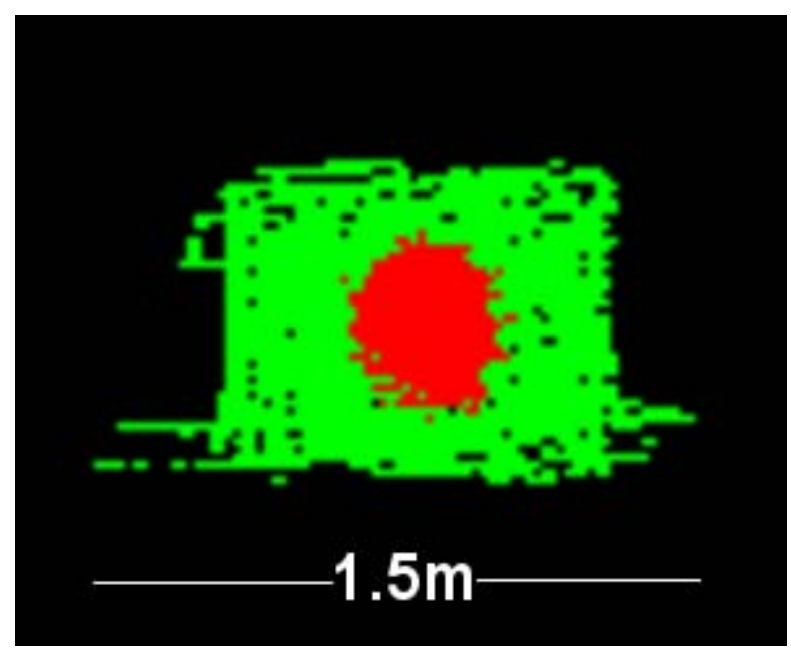

Figure 9. A thresholded radiation map of the MiRTLE system at $7.5 \mathrm{~m}$ without a horn antenna to increase receiver sensitivity

\section{CONCLUSION}

A cost sensitive implementation of the 'radiation camera' idea [6] element has been described to measure and map radiation in a user defined plane. Using readily available commercial off the shelf components the ability to measure and display radiation intensity, in real time, at video refresh rates, has been demonstrated without the need for an anechoic environment. The prototype system is handheld and manually manipulated by the user who relies on the real time mapping visible in the GUI to guide the mapping process. The compact form factor and the ability to manipulate the system components by hand minimises cost and reliance on bulky mechanical scanners. The small and very portable nature of the PU and wireless IR camera coupled with a laptop to display the data represent a system costing less than $£ 50$, excluding detector, that can be rapidly deployed and used anywhere.

\section{ACKNOWLEDGMENT}

The author would like to thank Chris Johnston for advice on methods of object tracking and David Andrews for his assistance taking experimental data.

\section{REFERENCES}

[1] M.J. Southgate "Remote detection of concealed guns and explosives", Manchester Metropolitan University, Manchester, 2013.

[2] QinitiQ (2012). QinetiQ Technology Showcase - New Technology Products from QinetiQ. [Online]. 2012. SPO-20 Technology Showcase. Available from:

http://technologyshowcase.qinetiq.com/. [Accessed: 25 May 2012].

[3] Kennedy, L. (2010). ThruVision Status 2010. [Online]. January 2010. Available from: http://www.etnuk.com/DesktopModules/Bring2mind/DMX/Download.aspx?Com mand=Core_Download\&EntryId=800\&PortalId=0\&TabId=2200. [Accessed: 1 June 2012].

[4] A. Agurto, Y. Li, G.Y. Tian, N. Bowring, and S. Lockwood, "A review of concealed weapon detection and research in perspective", in Proc. IEEE International Conference on Networking, Sensing and Control, 2007, pp. 443-448.

[5] Salmon, Neil A., Nick Bowring, Simon Hutchinson, Matthew Southgate, and Dean O'Reilly. "An aviation security (AVSEC) screening demonstrator for the detection of non-metallic threats at 28-33 GHz." In Society of Photo-Optical Instrumentation Engineers (SPIE) Conference Series, vol. 8900, p. 04. 2013.

[6] M.J. Southgate "Real time spatial characterisation of practical radio frequency sources", in MMU Science and Engineering Conference, 2013.

[7] "Emgu CV: OpenCV in .NET (C\#, VB, C++ and more)". [Online]. Available: http://www.emgu.com/wiki/index.php/Main_Page. [Accessed: 27Feb-2013].

[8] "OpenCV", SourceForge. [Online]. Available: http://sourceforge.net/projects/opencvlibrary/. [Accessed: 27-Feb2013].

[9] B. Peek, "Managed library for Nintendo's Wiimote". [Online]. Available: http://wiimotelib.codeplex.com/. [Accessed: 01-Mar2013].

[10] H. Bay, T. Tuytelaars, and L. V. Gool, "SURF: speeded up robust features," in ECCV (1), 2006, pp. 404-417.

[11] Bowring, Nicholas J., Matthew J. Southgate, David A. Andrews, Nacer D. Rezgui, Stuart W. Harmer, and Dean O'Reilly. "Development of a longer range standoff millimetre wave radar concealed threat detector." In SPIE Defense, Security, and Sensing, pp. 87140C-87140C. International Society for Optics and Photonics, 2013. 